\title{
Challenges in Delivering Tele-Practice Services for Communication Disorders Among Audiologists and Speech Language Pathologists
}

\author{
Biraj Bhattarai $^{1}$ - Tanvi Sanghavi ${ }^{1}$ (D) B. P. Abhishek ${ }^{2}$
}

Received: 21 August 2021 / Accepted: 7 December 2021/Published online: 14 January 2022

(C) Association of Otolaryngologists of India 2021

\begin{abstract}
Outbreak of COVID-19 pandemic has affected immensely the service delivery to patients with communication disorders. Tele-rehabilitation has become a new norm for the service in the field of speech and hearing. Daily challenges in service delivery are widely being reported. The objective of study is to highlight challenges faced by Audiologists and Speech Language pathologists during tele-practice. A descriptive cross-sectional survey was carried out through a web-based questionnaire regarding challenges faced by Audiologists \& Speech Language Pathologists, and their perspectives towards telepractice was compared. Total of 18 questions were divided into three sections. Section I included questions regarding training related to tele-practice, types of cases being handled, etc. Section II comprised of questions related to teletherapy resources and section III had questions related to evaluation and treatment by tele-mode. 118 participants (47\% Postgraduate students, 29\% undergraduate students and $24 \%$ Professionals) participated in the study. Only $16.1 \%$ of the clinicians were trained formally for service delivery via tele-mode. All the participants reported child language disorders, difficult to handle during sessions. Participants reported lack of evidence based tele resources for the use during their practice and also stated difficulty assessing and intervening clients via tele-mode. Results show telerehabilitation is being widely used. Challenges faced by Audiologists and Speech language pathologists are difficulty handling paediatric population, lack of
\end{abstract}

Tanvi Sanghavi

tanvisanghavi@gmail.com

1 All India Institute of Speech and Hearing, Mysore 570006, India

2 Nitte Institute of Speech and Hearing, Mangalore, India evidence-based tele-resources, and difficulty in evaluation $\&$ management of the patients. Effective training regarding tele practice is the need of current scenario. Future research on developing evidence- based resources is emphasized.

Keywords Tele-practice Challenges .

Communication disorders - Assessment and intervention . Evidence-based resources · Quality service

\section{Introduction}

Technological improvements and the development of many types of communications networks have resulted from innovation. Tele health, a part of such advancements, is being used by a growing number of healthcare providers. The term telemedicine refers to diagnostic and treatment services provided by a physician, nurse, or other healthcare professional across a long distance [1]. Audiologists and Speech language pathologists are one such group of healthcare professionals who provide tele services for a wide range of patient population. Tele-practice allows to give diagnostic and intervention services to a variety of patients with communication disorders over long distances using online videoconferencing systems and other dedicated telecommunication systems.

According to ASHA (2005a), telecommunication is defined as "The application of telecommunications technology to deliver professional services at a distance by linking clinician to client, or clinician to clinician for assessment, intervention, and/or consultation,". Tele-practice has a number of advantages, including increased access to speech-language pathology and audiology services, improved access to services for clients with linguistic and cultural diversity, increased ease of collaboration among 
multidisciplinary team members, and cost savings for clients [2]. The terms tele-practice and tele therapy will be used interchangeably in this study.

With the recent pandemic of COVID 19, social distancing has become the new norm. To prevent the spread of the disease, it is practised all over the world. This not only minimises the risk of transmission, but also could be useful for patients who cannot attend clinics regularly due to the unavailability of services near their reach [3].As a result, the possibilities of technology have been explored in practically every facet of life. Tele rehabilitation is the use of information and communication technologies (ICT) to give rehabilitation services to persons in their homes or other settings across a long distance [4]

There have been many studies debating over whether tele therapy is feasible and convenient over face-to-face therapy and the results have been found to be mixed [5-9]. Face-to-face therapies have long been regarded as the 'gold standard' for the treatment of patients with speech, language, and swallowing disorders, but tele therapy has emerged as a viable alternative for patients with a good educational and technological background [5]. Another study shows a higher level of acceptance and uptake of tele-practice, which was a pressing need at the time. Despite the fact that it appeared to be stressful to fast adapt to using tele-practice, the Speech Language Pathologists were quick to adapt and use the available technology to deliver services [8]

There are few speech pathologists and audiologists in India involved in tele-practice, even fewer who are trained to deliver services through tele-practice. The organisations recognised by Rehabilitation Council of India (RCI) can hold tele rehabilitation courses in many countries to raise the understanding of speech therapists about the technology, and research on the topic should also prioritised. It will also enhance therapists' attitudes regarding tele-practice and provide them with new information and abilities. These organisations must also pursue legal action in order to legitimise their services. Overall, it is proposed that professional groups take additional steps to remove hurdles in the way of therapists and promote tele-practice facilitators so that SLPs employ this sort of therapy more frequently [6].

Preliminary studies have been conducted to explore the professionals who have undergone any form of training, extensive research is needed to promote the idea of professionals being trained [10]. Another challenge is lack of evidence based and standardized resources for teletherapy with limited use of hands-on activities. This limitation can be extended to further lack of resources relevant for the Indian context. There is a need to explore the current practice patterns of the professionals and students delivering tele-practice services regarding use of resources.
To summarize, tele-practice in India poses unique challenges for the Audiologists and Speech language pathologists owing to the multilingual and multicultural issues, lack of evidence-based resources and lack of training of professionals. The present study aims to compare the practice patterns of professionals and students delivering tele-practice services for three specific situations: opinion regarding face-to-face therapy and tele therapy, source of resources and use of tele therapy during evaluation and treatment of various speech and language disorders.

\section{Method}

A descriptive cross-sectional study was performed with undergraduate, graduate-level students and professionals in the field of speech and hearing in India. Both male and female students and professionals, experienced with tele rehabilitation service were recruited for the study.

\section{Design of Questionnaire}

A survey questionnaire was prepared regarding the experience in practicing with tele-mode, confidence in exploring and using resources for tele-practice and challenges during evaluation and intervention with tele-practice, among the students and professionals of speech and hearing. The study authors organised the survey questionnaire with different demographic details, details related to experience, caseload, difficulty during tele-practice, telepractice resources and challenges during assessment and treatment in various setup. Three speech language pathologists with at least one year of experience with tele rehabilitation validated the questions. The necessary corrections suggested by the experienced professionals were incorporated during the finalization of the survey questions.

The self-assessment questionnaire consisted of 20 questions out of which 4 were related to the demographic details, 7 were related to experience with tele-practice and types of case load, 9 questions related to challenges faced during tele-practice. Challenges in finding evidence-based therapy resources, less responsiveness from client and caregiver along with difficulty during assessment and intervention, were questions included for the survey. All the questions were designed as objective having multiple choice.

The final version of the questionnaire was prepared in the e-survey in the form of google forms distributed through various social platforms by the researchers through convenient sampling. All the participants not responding to the survey were excluded from the study. 


\section{Informed Consent and Ethical Guidelines}

Ethical guidelines formulated by the institutional board of All India Institute of Speech and Hearing (AIISH), Mysore, were followed for the study. Informed consent was taken from all the participants in the form of a question in google form itself.

\section{Statistical Analysis}

The data obtained was subjected to statistical analyses using IBM Statistical package social sciences (SPSS) version 25.0 (SPSS Inc., Chicago). Results were analysed descriptively.

\section{Results}

A total of 118 participants (56 postgraduate students, 34 Undergraduate students and 28 audiologists/or speech language pathologists) responded to the survey. $77.6 \%$ of the participants were practicing at institutional setup followed by $13.6 \%$ of them providing service from private clinic. Rest $9.3 \%$ were linked up with hospitals. On assessment of the experience with tele-practice, $97.5 \%$ had reported to have experience of less than five years while rest $2.5 \%$ responded as having experience of more than 5 years. When asked about any form of training received by the participants, only $16 \%$ reported positively.

Patient's category working with along with difficult to handle patient group by the participants was assessed. Table 1 shows the age group working with by our participants and Table 2 depicts the percentage of participants reporting difficult to handle different patient group.

Types of communication disorder being served by the practitioner were asked with an open-ended question. Multiple responses from participants were obtained. Various disorders reported includes child language disorders (CLD), adult language disorders (ALD), motor speech disorders(dysarthria), fluency disorders, speech sound disorders (SSD), voice disorders (VD), and swallowing

Table 1 Age group worked with by the participants

\begin{tabular}{ll}
\hline Age group (years) & Number of participants \\
\hline $0-5$ & $104(88.1 \%)$ \\
$5-10$ & $84(71.2 \%)$ \\
$10-20$ & $42(35.6 \%)$ \\
$20-50$ & $41(34.7 \%)$ \\
$50-70$ & $26(22 \%)$ \\
Above 70 & $10(8.5 \%)$ \\
\hline
\end{tabular}

Table 2 Difficult to handle patient groups reported by participants

\begin{tabular}{ll}
\hline Difficult to handle patient group & Number of participants \\
\hline Children & $102(86.4 \%)$ \\
Adult & $3(2.5 \%)$ \\
Geriatric & $15(12.7 \%)$ \\
None & $10(8.5 \%)$ \\
\hline
\end{tabular}

disorders. Figure 1 shows the percentage of participants handling cases of various disorders. Similarly, a question with specifically focused into whether they face difficulty taking therapy for any of the cases was asked and $67.5 \%$ responded to struggle during therapy. An open-ended question to name three disorders difficulty in dealing with was enquired. All the participants reported child language disorders specifically those with behavioural issues like autism spectrum disorder (ASD), attention deficit hyperactivity disorder (ADHD), hearing impairment (HI) and more, were difficult to take treat online.

Participants perspective on the reduction on number of case load was assessed using 5-point Likert scale, their primary responses were 3.27 , where 5 indicating strong agreement and 1 indicating strong disagreement which might be suggesting participants did not find a difference in the caseloads when compared to face-to-face offline therapy. Similarly, 5-point Likert scale was used to assess clients' interest towards rehabilitation from participants view. The mean response for the question was 2.57 suggestive of decreased interest in attending therapy through tele-mode.

The second section of the questionnaire tried to assess perception of respondents regarding tele therapy resources and comprised of three questions, specifically focusing into difficulty in choosing therapy resources followed by

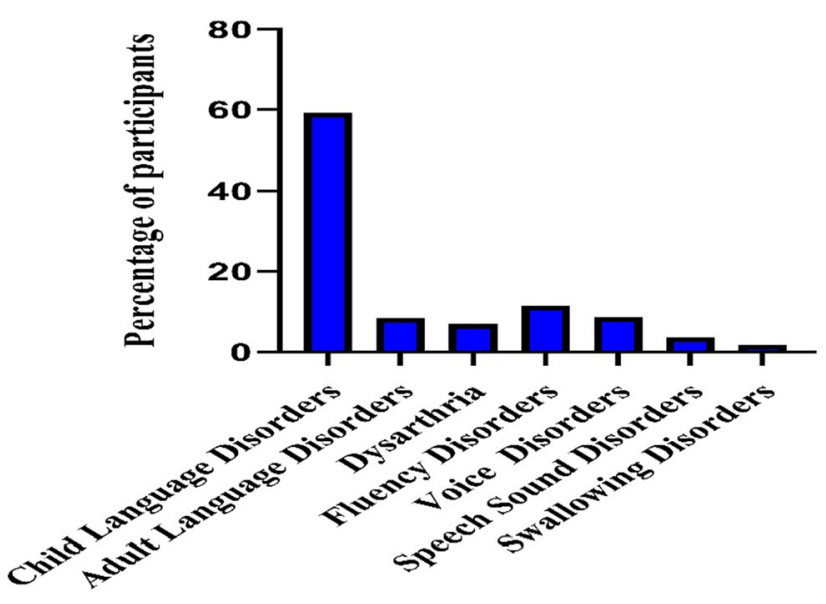

Disorders handled

Fig. 1 Percentage of participants handling various disorders 
evidence of the available therapy resources, and evidence of resources in the Indian context. All the questions were rated on 5-point Likert scale, where 5 indicated strong agreement and 1 suggestive of strong disagreement. Average responses of all the participants for three questions were found to be 3.2 which might suggest they might have no difficulty selecting therapy resources, 3.62 indicating participants may be into the line of agreement regarding lack of evidence and 4.11 suggesting participants do agree about no evidence- based resources in Indian context, respectively. An open-ended question was included to find out the source of therapy resources for telepractice. Most of the participants (71.18\%) reported they depend on various freely available online websites while remaining participants responded as they would tailor made therapy material for the clients by themselves or use multimedia like PowerPoint, YouTube or involve parents for therapy.

Final section included statements related to challenges during evaluation procedures and intervention. Total of three statements, all rated on 5-point Likert scale, with 5 being strongly agree and 1 being strongly disagree, were presented to participants which included difficulty assessing the client in absence of caregiver, possibility in achieving the goals via tele-mode and difficulty in training caregiver. Mean responses for three statements were obtained. The mean average responses obtained were observed to be 4.11 which suggests participants agreeing on need of caregiver for assessment, followed by 2.94 indicative of respondents might not have found differences in achieving goals through tele-mode, and 3.17 on training caregivers suggestive of not adequate confidence in training caregivers to follow home program, respectively.

\section{Discussion}

The rapid spread of COVID-19 pandemic has made many healthcare professionals stick to tele-practice to deliver services and reach patients which could not be done with face-to-face therapy. The present study aimed to compare practice patterns of professionals and students working in the field of audiology and speech language pathology. Majority of the participants who responded to the survey were postgraduate students followed by undergraduate students and working audiologists and speech language pathologists. This might be due to the high number of caseloads assigned to postgraduate students in an institutional setup as compared to other groups of participants.

Only around $16 \%$ of the participants reported to receive some form of training to deliver services through telepractice. The main reason for this low percentage of manpower getting trained in tele-practice is lack of such formal training to the students during their training period. Moreover, there lacks such provision in the academic curriculum in speech and hearing. Mohan et al. (2017) reported that it was deemed necessary to have at least a degree of Masters in Audiology or Speech language pathology to receive certification of delivering tele-practice services.

Most of the participants worked with paediatric age group, followed by adults and geriatric population as incidence and prevalence of paediatric cases of communication disorder is higher. Similar findings were reported in a study by [8]. The cases which were difficult to handle during tele-sessions were mainly the paediatric group followed by geriatric and adult group. Within paediatric group most of the participants reported child language disorders such as autism spectrum disorder (ASD), attention deficit hyperactivity disorder (ADHD) and Hearing Impairment (HI). This is mainly to do with the behavioural issues these child exhibit along with difficulty comprehending the instruction provided and expressing the need as per the context. Also, many of these children do present with reduced intellectual functioning that adds more into the pre-existing problem. Interestingly, a similar trend was observed in a study and reported that children with these behavioural disorders are difficult to handle [4]. Other reasons for difficulty managing ASD and ADHD cases could be due to lack of adequate pre-linguistic skills due to hyperactivity and inattention, which makes sitting in front of the computer and following the clinician's directions challenging. One of the most difficult obstacles for SLPs was managing children online, followed by a lack of internet resources concerning tele rehabilitation and parents' or patients' unwillingness to switch from traditional face-to-face therapy to tele rehabilitation [4].

In the present study, the participants did not find a difference in providing services via tele-practice as compared to face-to-face therapy, however they did report decreased client interest in attending therapy via tele-practice. Such finding could mean clinicians are now comfortable providing services through tele-practice. Supporting our findings, Rangaswamy and Rao [11] also reported tele-practice being embraced quite positively by speech language pathologists for service delivery. But as discussed earlier the behavioural issues in children could lead to decrease in attention span and difficulty following the instructions given, and stimuli presented via online mode reducing the effectiveness of service delivery. Also due to lack of cooperation of the client, caregivers also might not feel interested for the online session, thereby possibly questioning the effectiveness of tele-rehabilitation.

The next section of the questionnaire aimed to assess the opinion of the participants regarding use of resources for tele-practice. Majority of them did not find difficulty in 
selecting resources suggesting that there are resources available online. With increase in digitization, it is quite easy to search for the desired resource through websites. However, lack of evidence-based resources globally and also in Indian languages were the challenges faced by our participants. It points to the fact that there is dearth in the research done in the area of tele-practice [8]. More students and professionals need to be involved in research practice to understand the needs of the stakeholders and devise resources accordingly. The resources need to be culturally and linguistically appropriate especially in a multilingual country like India. When participants were asked to mention the resources used during tele-practice, wide variety of options were mentioned such as Pinterest, online websites, self-made resources, teachers pay teachers etc. Therefore, there is a lack of consensus among the speech and hearing professionals for use of tele therapy resources. This uneven choice of resources is due to lack of monitoring and appropriate guidelines framed for tele-practice by the governing body (Rehabilitation Council of India (RCI) and Indian Speech and Hearing Association (ISHA)) in the field.

The last section of the questionnaire aimed to find challenges during evaluation and treatment of speech and language disorders. It was observed that there is a need for the presence of caregiver during most sessions to explain the goals and follow the home program for the patient. Need of a caregiver during evaluation and intervention is because there are various types of tasks given during this process which needs constant monitoring and prompts from the caregivers. In absence of clinician with the client faceto-face it is most for the caregivers to be with the client throughout the session of assessment and intervention.

A positive response was seen with respect to achievement of the goals set for the patient compared to face-toface therapy. This could be due to better communication between clinician and caregivers, increased participation of caregiver in the rehabilitation program, increased accessibility, sharing of online resources and possibly increased frequency of teletherapy sessions. Audiologists and speech language pathologist must identify whether clients or caretakers are comfortable with computers and technology, which are required for tele-practice execution. Whether child clients can use equipment related to tele-practice services and whether their cognitive function or motor ability is adequate to receive speech [12]. If the aspects deemed challenging for the caregivers are taken care of, acceptance of tele-practice can improve even more and better results could be obtained with regards to evaluation and treatments. Therefore, this study gives interesting insights of tele-practice use among the students and working professionals of speech and hearing.

\section{Conclusion}

This study clearly highlights the challenges faced by the practicing clinicians in the field of speech and hearing. Majority of the participants did not receive any formal training. Most of them reported paediatric cases to be more challenging to handle during the tele mode of service delivery. Lack of evidence-based therapy resources was another challenge faced by the participants in our study. Therefore, there is need of formal training to the professionals to use the tele service efficiently. This can be done by introducing the guidelines of tele-practice into the curriculum, so that this will provide students with sound knowledge and practical skills and help them boost their confidence in handling cases of any kind. The skill sets regarding tele-practice are immense, and this study highlights the gaps in confidence in performing these skills in the time of need by speech and hearing students and professionals. This study further strongly recommends incorporating adequate training in tele-rehabilitation as part of the speech and hearing course curriculum. Hence, it is highly recommended to the stakeholders to look into this aspect in the future so that competent human resources are produced from their institute that could provide service in the field of speech and hearing with no compromise. Also, future studies on developing evidence-based therapy resources in Indian languages is suggested to provide a quality service to needy.

Acknowledgements The authors acknowledge with gratitude Department of Audiology and Speech Language Pathology, All India Institute of Speech and Hearing for permitting to conduct the study at the institute. The authors also like to acknowledge the participant for co-operation.

Authors' Contribution BB was involved in study design, stimulus preparation, data collection, analysis of the data, interpretation and writing the manuscript; TS was involved in study design, stimulus preparation, data collection, analysis of the results, interpretation and writing the manuscript; and ABP was involved in concept development, study design, analysis of the results and writing the manuscript.

Funding There is no funding by any agency for the manuscript.

\section{Declarations}

Conflict of interest The authors report no conflicts of interest. The authors alone are responsible for the content and writing of the paper.

Ethical Approval All procedures performed in this study were in accordance with the ethical guidelines of bio-behavioral research involving human subjects of the All India Institute of Speech and Hearing, Mysore.

Ethical Standards The manuscript adheres to the ethical standards according to the Declaration of Helsinki. 
Informed Consent Informed consent was obtained from the patient to participate in the study.

\section{References}

1. Fong B, Fong ACM, Li CK (2010) Telemedicine technologies: information technologies in medicine and telehealth. John Wiley \& Sons Ltd, Chichester

2. Theodoros DG (2008) Telerehabilitation for service delivery in speech-language pathology. $\mathrm{J}$ Telemed Telecare. https://doi.org/10.1258/jtt.2007.007044

3. Bhatarai P, Bhattarai B, Karna SL (2020) Audiology and speechlanguage pathology services in pediatric population during COVID Pandemic in Nepal. Nepal Med J 3:398-401. https://doi.org/10.3126/NMJ.V3I2.32325

4. Vrinda R (2020) Telerehabilitation in the field of speech language pathology during pandemic Covid19 outbreak-an analysis in Kerala. Biosci Biotechnol Res Commun 13:2281-2288. https://doi.org/10.21786/bbrc/13.4/99

5. Chaudhary T, Kanodia A, Verma $\mathrm{H}$ et al (2021) A pilot study comparing teletherapy with the conventional face-to-face therapy for speech-language disorders. Indian J Otolaryngol Head Neck Surg. https://doi.org/10.1007/s12070-021-02647-0

6. Tohidast SA, Mansuri B, Bagheri R, Azimi H (2020) Provision of speech-language pathology services for the treatment of speech and language disorders in children during the COVID-19 pandemic: problems, concerns, and solutions. Int J Pediatr Otorhinolaryngol. https://doi.org/10.1016/j.ijporl.2020.110262

7. Macoir J, Sauvageau VM, Boissy P et al (2017) In-home synchronous telespeech therapy to improve functional communication in chronic poststroke aphasia: results from a quasiexperimental study. Telemed e-Health 23:630-639. https://doi.org/10.1089/tmj.2016.0235

8. Aggarwal K, Patel R, Ravi R (2020) Uptake of telepractice among speech-language therapists following COVID-19 pandemic in India. Speech, Lang Hear. https://doi.org/10.1080/2050571X.2020.1812034

9. Valentine DT (2015) Stuttering intervention in three service delivery models (direct, hybrid, and telepractice): two case studies. Int J Telerehabil. https://doi.org/10.5195/ijt.2014.6154

10. Mohan HS, Anjum A, Rao PKS (2017) A survey of telepractice in speech-language pathology and audiology in India. Int $\mathbf{J}$ Telerehabil. https://doi.org/10.5195/ijt.2017.6233

11. Rangaswamy Y, Rao P (2018) Tele speech-language pathology and audiology in India- a short report. J Int Soc Telemed EHealth. https://doi.org/10.29086/JISfTeH.6.e19

12. Yoo J, Yoon MS, Lee CK et al (2020) An exploratory survey of priorities in establishing telepractice system for SLPs and caregivers in Korea. Commun Disord Q. https://doi.org/10.1177/1525740120916414

Publisher's Note Springer Nature remains neutral with regard to jurisdictional claims in published maps and institutional affiliations. 